\title{
Factors Affecting the Outcome of a Pulmonary Resection for Metastatic Colorectal Cancer: Efficacy and Pitfalls
}

\author{
Ik Yong Kim \\ Department of Surgery, Yonsei University Wonju College of Medicine, Wonju, Korea
}

See Article on Page 354-358

Pulmonary resection of metastatic colorectal cancer is widely practiced in the field of oncologic surgery. However, only a highly selected subset of patients is eligible for resection, and the average recurrence rate is still high. According to general guidelines proposed by the UK National Institute for Clinical Excellence [1] and the National Comprehensive Cancer Network [2], however, for the following cases of metastatic pulmonary lesions, surgery is indicated:

1) the metastases seem to be technically resectable,

2) the general and functional risks are tolerable,

3) the primary tumor is controlled, and

4) no extrathoracic lesions are detected (with the exception of hepatic lesions for which both the hepatic and the pulmonary metastases can be completely removed).

According to a review of the literature on surgical management of a pulmonary metastasis of colorectal cancer, the results vary depending on the selection criteria of individual surgeons or institutions. Due to the problems associated with a lack of randomized, prospective clinical studies, it remains difficult from an evidence-based perspective to assess or summarize the clinical usefulness of surgical management for metastatic lesions [3]. To date, however, most of the retrospective studies have demonstrated that a surgical resection of the pulmonary metastasis of colorectal cancer is a safe treatment modality with a lower degree of mortality.

The following meta-analysis for a pulmonary metastasectomy in cases of colorectal cancer showed that for all 1,307 patients investigated, a minimum number of 40 cases with a mean fol-

Correspondence to: Ik Yong Kim, M.D.

Department of Surgery, Yonsei University Wonju College of Medicine,

Heungeop-myeon, Wonju 220-710, Korea

Tel: +82-33-741-0573, Fax: +82-33-744-6604

E-mail: iykim@yonsei.ac.kr

(C) 2010 The Korean Society of Coloproctology

This is an open-access article distributed under the terms of the Creative Commons Attribution NonCommercial License (http://creativecommons.org/licenses/by-nc/3.0) which permits unrestricted noncommercial use, distribution, and reproduction in any medium, provided the original work is properly cited. low up of 37.6 months, the overall 5-year survival was 40-68\% after complete surgical resection, and the mean 5-year diseasefree survival after complete resection was 19.5-34.4\%. Recent reports reflect better treatment outcomes, but not come to the of all the patients [4]. Traditional risk factors and selection criteria are currently being discussed, but decision making presupposes a personalized strategy with predictive models. Such predictive models have a number of uses. In particular, due to the selection of patients who might benefit from such treatment modalities as neoadjuvant chemotherapy prior to a prethoracotomy, surgery, or active chemotherapies, those with good prognostic factors can receive the surgical treatment at earlier times and those with a poor prognosis are subject to delayed surgery. This delay, or "trial of time," can allow a better assessment of additional disease and provide time for neoadjuvant therapy or more aggressive adjuvant therapy. Further, it can also be used to enroll patients who are at increased risks of recurrence in prospective randomized trials and can provide a postoperative surveillance strategy following the actual surgery [5].

On a multivariate analysis, Kanemitsu et al. [6] maintained that five prognostic factors, the primary histology, hilar or mediastinal lymph node involvement, the number of metastases, the preoperative level of the carcinoembryonic antigen, and extrathoracic disease, would be risk factors. These factors have a prognostic value for long-term survival, and related study models are advantageous in that the degree of clinical applicability is relatively higher. In addition, other prognostic factors affecting the survival rate following the pulmonary resection include a disease-free interval during which a diagnosis of pulmonary lesions is made. As shown in this study, however, there was no significant difference.

In recent years, as a prognostic factor for pulmomary metastasis, genetic tests based on such technologies as the Affymetrix Gene Chip microarray have been applied. However, because of the prohibitive costs, reliance on frozen tissue, and advanced technical expertise required to use the technology, it is debatable whether such tests will ever be clinically applicable [7]. Thus, the immunohistochemical (IHC) staining profile can be a useful surrogate for gene expression analysis. Many studies 
have focused on the correlation between histopathologic and IHC measurements and survival $[8,9]$. According to Shiono et al. [9] the histopathologic features of pulmonary metastases in 87 patients revealed the morphologic feature of an aerogenous spread with floating cancer cell clusters and vascular invasion at metastatic sites to be a prognostic factor of significance for survival. Lymphatic invasion or microsatellite instability of the primary tumor, however, has been reported to be unrelated to survival. Although smaller in number, with the recent advancement in molecular biological methods, ongoing studies are being conducted to examine the pulmonary metastasis of colorectal cancer. These studies will provide us further understanding of pulmonary metastatic cascade and allow patient treatment to be personalized.

\section{REFERENCES}

1. National Institute for Clinical Excellence. Improving Outcomes in Colorectal Cancers: guidance on cancer services. London: National Institute for Clinical Excellence; 2004 [cited 2010 Oct 18]. Available from: http://www.nice.org.uk/nicemedia/pdf/CSGCCfullguidance.pdf.

2. National Comprehensive Cancer Network. NCCN Clinical Practice Guidelines in Oncology (NCCN Guidelines). Washington, PA: National Comprehensive Cancer Network; c2010 [cited 2010 Oct 1]. Available from: http://www.nccn.org/professionals/phy- sician_gls/f_guidelines.asp.

3. Treasure T. Pulmonary metastasectomy: a common practice based on weak evidence. Ann R Coll Surg Engl 2007;89:744-8.

4. Pfannschmidt J, Hoffmann H, Dienemann H. Reported outcome factors for pulmonary resection in metastatic colorectal cancer. J Thorac Oncol 2010;5(6 Suppl 2):S172-8.

5. Treasure T, Fallowfield L, Farewell V, Ferry D, Lees B, Leonard P, et al. Pulmonary metastasectomy in colorectal cancer: time for a trial. Eur J Surg Oncol 2009;35:686-9.

6. Kanemitsu Y, Kato T, Hirai T, Yasui K. Preoperative probability model for predicting overall survival after resection of pulmonary metastases from colorectal cancer. Br J Surg 2004;91:112-20.

7. Wuttig D, Baier B, Fuessel S, Meinhardt M, Herr A, Hoefling C, et al. Gene signatures of pulmonary metastases of renal cell carcinoma reflect the disease-free interval and the number of metastases per patient. Int J Cancer 2009;125:474-82.

8. Kayser K, Zink S, Andre S, Schuring MP, Hecker E, Klar E, et al. Primary colorectal carcinomas and their intrapulmonary metastases: clinical, glyco-, immuno- and lectin histochemical, nuclear and syntactic structure analysis with emphasis on correlation with period of occurrence of metastases and survival. APMIS 2002;110:435-46.

9. Shiono S, Ishii G, Nagai K, Murata Y, Tsuta K, Nitadori J, et al. Immunohistochemical prognostic factors in resected colorectal lung metastases using tissue microarray analysis. Eur J Surg Oncol 2006;32:308-9. 